\title{
Analysis on Influencing Factors of Services Satisfaction with Family Doctors and Contract Signing----Take Hangzhou as an Example
}

\author{
Tao Fang ${ }^{1} \quad$ Songyan Zhang ${ }^{1 *} \quad$ Lichao Tao $^{2}$ \\ 1.School of Economics and Management, Zhejiang University of Science and Technology, Hangzhou \\ 310023, China \\ 2.School of Management, Zhejiang University, Hangzhou 310058,China
}

This research is financed by the 2019 Zhejiang University of Science and Technology extracurricular Science and Technology Innovation and Practice project; Science and Technology Innovation Activity Plan for Zhejiang province University Students in 2020 (Grant No.2020R415022);Innovation and Entrepreneurship Training Program for University Students of Zhejiang University of Science and Technology in 2020(Grant No.2020CXCY033); National Innovation and Entrepreneurship Training Plan for College Students in 2020(Grant No.202011057033).

\section{Abstract}

Family doctors, as an important part of primary medical care, are the cornerstone for the full implementation of the Healthy China policy and the realization of health for all in 2030. In order to understand the current situation of family doctor service and contract signing in Hangzhou, on the basis of a questionnaire survey with residents in various districts of Hangzhou, the importance matrix was adopted to analyze the satisfaction of contracted residents, principal component analysis and binary logistic regression were used to explore the influencing factors of signing the contract, it has been found that the service level, medical guidance, derivative services and future development significantly influenced the residents' intention to sign contracts. According to the research and analysis, some suggestions have been proposed to further improve the family doctor policy.

Keywords: Family doctor; Medical services; Satisfaction; Contract situation

DOI: $10.7176 / \mathrm{JESD} / 11-20-01$

Publication date:October $31^{\text {st }} 2020$

\section{Introduction}

Family doctors, as an important part of primary medical care, play a role as a link between the community and higher-level hospitals. With family doctors as the connection point, the establishment of a diagnosis and treatment pathway from the community-first to two-way referral will further improve the tiered diagnosis and treatment mode, and achieve the purpose of improving the operation efficiency of the medical and health system and saving public medical resources. Meanwhile, family doctors can provide various medical and health care services including round-the-clock consultation, online consultation and rehabilitation guidance for contracted residents, effectively meeting the daily medical needs of residents and prominently relieving the pressure on primary medical care. The Medical Reform Office of the State Council jointly launched the Guiding Opinions on Promoting Family Doctors' Contract Services with six departments in June 2016, which clearly pointed out that the contracting of family doctors will be accelerated across the country on the basis of some pilot projects. The Healthy China policy put forward in the report of the 19th National Congress of the Communist Party of China also regards family doctors as the focus of policy implementation. The contract system of family doctors has become a key link in the health of the public. Therefore, it is of certain practical significance to explore the development of the family doctor system.

Scholars at home and abroad have conducted numerous researches on family doctors in different aspects. From the perspective of the efficiency and motivation of family doctors, Lapcevic $\mathrm{M}$ et al. (2006) have found that work enthusiasm plays a decisive role in the service efficiency of family doctors after investigating 828 general practitioners. Peifang Wang et al. (2018) believe that inconsistent behaviors between family doctors and residents will reduce their efficiency from the perspective of incentive compatibility theory. On the relationship between family doctors and tiered diagnosis and treatment system, Liqun Liu (2018) believes that there are still problems of unbalanced work development and insufficient basic medical capacity in China. Bindman AB et al. (2003) point out that tiered diagnosis and treatment by family doctors simplifies the steps for patients to go to higher-level hospitals for medical treatment. Other scholars have studied the new direction of family doctors from the perspective of the Internet. For example, Albert SM et al. (2011) believe that online diagnosis and treatment based on informatization has brought new ways for family doctors to consult. Zhijie Song et al. (2018) have discussed the resource integration service system under the background of "Internet + Family Doctors". From a statistical point of view, Xueyan Zhang et al. (2020) have found that factors such as gender, annual income and medical expenses have a greater impact on the satisfaction of contracted residents in Jiangsu Province. Jiaoling Huang et 
al. (2019) have found that the cognition and satisfaction with family doctors' consultation and the location of the first diagnosis significantly impacted the willingness of residents in Hongkou, Shanghai to sign up for family doctors. However, existing researches seldom analyze the satisfaction of family doctors' contracted services and the influencing factors from the perspective of econometric empirical evidence. In particular, there is a lack of relevant research on Hangzhou's family doctor system. Therefore, this paper explores the satisfaction and influencing factors of the contract services of Hangzhou's family doctors through on-the-spot questionnaire survey and analysis.

\section{Analysis on the Development Status of Family Doctor Contract System in Hangzhou City}

Hangzhou, as the first city to carry out the pilot family doctor system, has formed a unique "Hangzhou Model" after nearly ten years of exploration since the four major directions of the new medical reform were drawn up in 2009. At present, the development of Hangzhou's family doctor contract system presents the following characteristics

\subsection{Rapid Development of the Contract System}

As a coastal economically developed city, the people of Hangzhou has shown a high degree of acceptance of the new system. In 2011, Hangzhou first conducted a pilot project of the family doctor contract system in Shangcheng District, and in 2014, under the guidance of the "integration of medical care" policy, it promoted the combination of family doctors and community diagnosis, guiding 250,000 citizens to sign contract with family doctors one by one within one month. In 2017, Hangzhou carried out a comprehensive promotion of the system in all districts. While continuously improving the coverage of the family doctor contract system, Hangzhou successively issued a number of policies to optimize the service system and improve the service quality of family doctors. At present, 3.04 million of Hangzhou's 10 million permanent residents have signed up for family doctors, with a signing rate of more than 30\%. In addition, although Qiantang New District was newly established in the second half of 2019, the number of family doctors contracted has also grown rapidly, with more than 40,000 people signed up since 2020 .

\subsection{Diversification of Contract Services}

With the gradual advancement of the pilot work, the family doctor service has also been continuously developed from the basic of community clinic, showing the characteristics of diversification and individuation. Derivate from "Internet + Family Doctors", Hangzhou has launched a special APP for family doctors, with which Citizens can make appointments for medical treatment and physical examination through mobile phone operation. After physical examination, they can check the results on mobile phones. For the elderly who are not familiar with the operation of mobile phones, they can also use the telephone to contact the doctor for consultation or make an appointment for door-to-door service. For patients with chronic diseases who need long-term medication, family doctors can issue long prescriptions to avoid the trouble that patients need to go to the hospital many times. Family doctors will also set up health records for each contracted resident, recording his or her physical information and medical treatment in detail, so as to provide personalized, long-term and systematic health guidance and ensure the health of residents during the contract period.

\subsection{Regional Imbalance of Contracting}

Although Hangzhou has witnessed a rapid development of the family doctor system and the signing rate has been greatly improved, it is found in actual survey that there is a big gap in the signing rate between different regions in the city. According to inquiries, 119,100 people signed contracts in Shangcheng District in 2019, with a signing rate of about $34.5 \%$, while 106,300 people signed contracts in Binjiang District, with a signing rate of $23.36 \%$. Although there is only a difference of 10,000 people between the two, the actual difference of signing rate is as much as $12 \%$. Judging from the horizontal comparison between communities, the situation is even more serious. In the actual visit, it is found that almost everyone has signed a contract in certain communities in the Shangcheng District. Comparing with the $34.5 \%$ signing rate in the whole area, it is not difficult to estimate the situation of other communities. This serious imbalance between regions will lead to a limited implementation of relevant policies in some districts or even in some streets, and will eventually make policies become a mere formality.

\section{Survey and Analysis}

In order to understand the satisfaction with family doctors in Hangzhou and the influencing factors of signing contracts, the method of questionnaire survey and field interview was adopted to conduct a survey in various districts of Hangzhou. The subjects of the survey are residents in Hangzhou. The questionnaire was designed from three aspects: awareness, satisfaction and demand. The potential variables of perception in satisfaction and demand were designed with the Likert scale, which is commonly used in the world. Questionnaires were issued according to the population proportion of all districts in Hangzhou and 572 questionnaires were collected, among which 42 
invalid and erroneous questionnaires were eliminated, and 530 valid questionnaires were eventually obtained, with an effective rate of $92.66 \%$. The following is an analysis of the survey results from three aspects: the basic conditions of the survey objects, the influencing factors of residents' willingness to sign contracts and the satisfaction of the contracted residents.

\subsection{Analysis of Basic Conditions of Survey Objects}

After sorting out and summarizing, the personal background and relevant information of the subject objects are shown in the following table 1:

Table 1.Analysis of the survey objects

\begin{tabular}{lll|lll}
\hline variable & options & percentage (\%) & variable & options & percentage $(\%)$ \\
\hline Sex & Male & 50.94 & Per capita & $<2000$ yuan & 4.15 \\
& Female & 49.06 & monthly & $2001 \sim 4000$ yuan & 22.45 \\
Age & $<30$ & 34.34 & income & $4001 \sim 6000$ yuan & 36.60 \\
& $30 \sim 45$ & 24.72 & & $6001 \sim 8000$ yuan & 16.98 \\
& $46 \sim 60$ & 18.87 & & $>8000$ yuan & 19.81 \\
& $>60$ & 22.08 & Health & Very poor & 0.94 \\
Census & Hangzhou area & 80 & status & Poor & 7.17 \\
register & Non-Hangzhou & 20 & & General & 27.74 \\
Whether or & Yes & 32.26 & & Relatively healthy & 46.23 \\
not to sign & No & 67.74 & & Very healthy & 17.92
\end{tabular}

As can be seen from Table 1, the distribution of the surveyed objects in the terms of the gender and age levels is relatively balanced, which shows that the selection of the questionnaire survey objects is reasonable with high credibility. Judging from the distribution of the per capita monthly income of the surveyed households, the overall range distribution is relatively reasonable, with the middle income of 4001-6000 accounting for 36.6\%, which is in line with the per capita monthly income of Hangzhou residents. Moreover, it can be seen from the table that the proportion of residents with a value of 6001-8000 yuan and more than 8000 yuan has reached about 36\%. At the same time, the comparison of data shows that the proportion of these people is relatively high of signing contracts, which shows that the improvement of living standards will promote people's attention to their own health status and further improve the signing rate of family doctors. In terms of health status, the health status of the surveyed subjects basically presents a normal distribution, among them, the number of residents who regard they are relatively healthy is the largest, accounting for $46.23 \%$. On the contrary, only $0.94 \%$ of the consider themselves in very poor health and they need to go to hospitals frequently. This shows that the physical condition of residents in Hangzhou area is generally good, which is also due to the promotion of the family doctor contract system. As far as the signing situation is concerned, the signing rate of the respondents is $32.26 \%$, which is also in good agreement with the overall signing rate of Hangzhou.

\subsection{Satisfaction Analysis of Contracted Residents}

In the analysis of the satisfaction of the contracted residents to the contract services, this paper uses the importance matrix to analyze the satisfaction considering the diversified characteristics of satisfaction indicators according to the results of Zhou Hang et al. (2018), and makes certain improvements to the measurement of importance, choosing the objective weight based on correlation as the importance indicator to measure. Firstly, the average value of each satisfaction index of the contracted residents is calculated and taken as a secondary index. Based on the second index, the arithmetic average is obtained to get the overall satisfaction of family doctors, which is taken as a first-level index. Finally, SPSS software is used to analyze the correlation between each second-level index and the first-level index, to extract the correlation coefficient and standardize it, and obtain the importance weight of each second-level index, that is, its importance in the overall satisfaction. The relevant data are shown in Table 2 .

Table 2 .Importance - Satisfaction related data

\begin{tabular}{cccc}
\hline Project & $\mathrm{r}$ & Importance & Satisfaction \\
\hline Medical charges & 0.765 & 0.108 & 3.813 \\
Medical level & 0.800 & 0.113 & 3.585 \\
Service attitude & 0.716 & 0.101 & 4.193 \\
Service effect & 0.851 & 0.12 & 3.632 \\
Service programs & 0.838 & 0.118 & 3.456 \\
Service team & 0.796 & 0.112 & 3.725 \\
Treatment time & 0.769 & 0.108 & 3.690 \\
Waiting time & 0.729 & 0.103 & 3.556 \\
Appointment referral & 0.844 & 0.119 & 3.485 \\
\hline
\end{tabular}


The importance-satisfaction matrix can be established on the basis of relevant data, as shown in Figure 1. It can be seen from the figure that there is only one program "service effect" in the first quadrant at present, which shows that for the contracted residents, the service effect is an indicator that they pay close attention to, and their satisfaction with the service effect is relatively high, which should be maintained and carried forward for family doctors. The programs in the fourth quadrant include "medical level", "appointment referral" and "service programs". For the contracted residents, these programs are rather important, but their satisfaction is relatively low, which indicates that family doctors are absent in these programs at this stage. Therefore, improvements should be made in these programs. The third quadrant is the secondary improvement point. The importance and satisfaction of programs in this filed are rather low, among which programs with higher importance should be improved first, such as "waiting time". The last is the second quadrant, the four factors of "service attitude", "medical charge", " treatment time" and "service team" in this field are relatively low in importance but high in satisfaction, which can be maintained continuously. However, also because of its low importance, the resources allocated to these programs can be appropriately allocated to those programs in the fourth quadrant on the basis of maintaining their original advantages to improve satisfaction of the programs of the fourth quadrant.

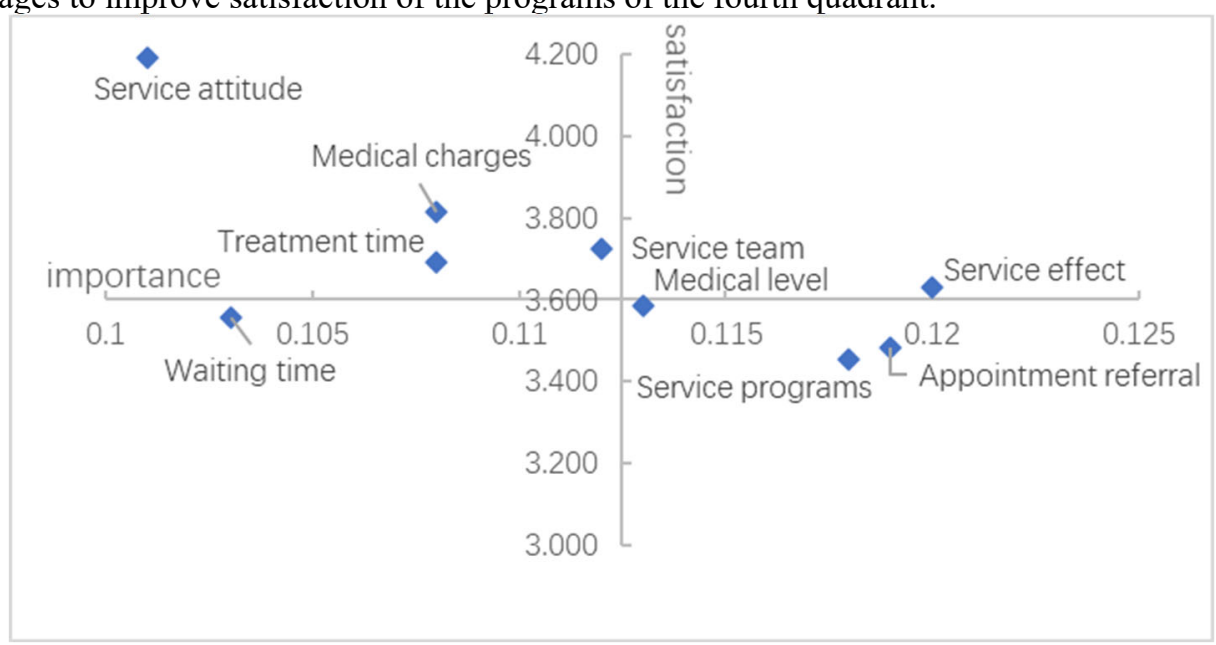

Figure 1. Importance-satisfaction matrix

\subsection{Analysis of Influencing Factors of Residents' Willingness to Sign up}

Before studying the influencing factors, reliability and validity should be used to analyze whether the sample answers are reliable and whether the questionnaire topic designed is reasonable, so as to judge the accuracy and stability of the questionnaire test results. On the basis of questionnaire survey, this paper uses KMO and Bartlett test to carry out validity analysis. It is found that $\mathrm{KMO}$ value is greater than 0.90 , which indicates that the statistics have strong partial correlation and are suitable for factor analysis. At the same time, according to Approx. ChiSquare of Bartlett's test, the significance probabilities are all 0.000 , less than $1 \%$, which indicates that the correlation between various variables is extremely strong, so factor analysis is effective. Then this paper uses principal component analysis to analyze the scale, and extracts five main factors about residents' contract signing: service level, medical guidance, derivative services, health management and future development. The reliability of the scale is tested by Cronbach's alpha coefficient on SPSS software, as shown in Table 3. The results show that the coefficient values of each variable are all greater than 0.7 , indicating that the scale involved in this survey has high internal consistency and can be used as survey factors.

Table 3 .Reliability analysis of factors

\begin{tabular}{cc}
\hline Dimensionality & Cronbach's Alpha value based on standardized items \\
\hline Service level & 0.925 \\
Medical guidance & 0.891 \\
Derivative services & 0.900 \\
Health management & 0.862 \\
Future development & 0.767 \\
\hline
\end{tabular}

Based on the results of reliability and validity analysis, this paper puts forward the following research hypotheses: Hypothesis 1: Service level has a significant impact on residents' willingness to sign up for family doctors.

Hypothesis 2: Medical guidance has a significant impact on residents' willingness to sign up for family doctors. Hypothesis 3: Derivative services have a significant impact on residents' willingness to sign up for family doctors. Hypothesis 4: Health management has a significant impact on residents' willingness to sign up for family doctors. Hypothesis 5: Future development has a significant impact on residents' willingness to sign up for family doctors. On the basis of the above hypotheses, considering whether signing a contract is a dichotomous variable, this paper 
uses a binary logistic model for fitting prediction, and constructs an equation based on the model in Fig. 2.

$$
\operatorname{Ln}\left(\frac{P}{1-P}\right)=\alpha+\beta_{1} X_{1}+\beta_{2} X_{2}+\Lambda+\beta_{n} X_{n}
$$

The dependent variable of the equation is "whether to sign a contract" and the independent variables are "service level", "medical guidance", "derivative service", "health management" and "future development". Combined with the survey data, SPSS 18 is used to fit the results, as shown in Table 4.

\section{Logistic}

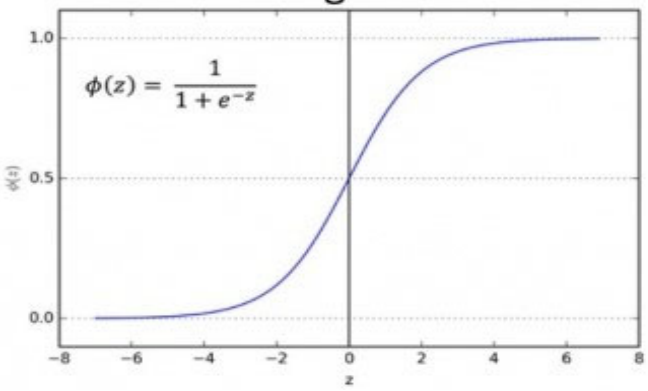

Figure 1 .logistic model

TABLE 4. LOGISTIC REGRESSION OF RESIDENTS' SIGNING INTENTION

\begin{tabular}{ccccccc}
\hline variable & B & S.E, & Wals & df & Sig. & Exp (B) \\
\hline Service level X1 & 0.742 & 0.336 & 4.859 & 1 & 0.028 & 2.100 \\
Medical Guidance X2 & -0.368 & 0.184 & 4.011 & 1 & 0.045 & 0.692 \\
Derivative service X3 & 0.656 & 0.279 & 5.530 & 1 & 0.019 & 1.927 \\
Health management X4 & -0.346 & 0.237 & 2.128 & 1 & 0.145 & 0.707 \\
Future development X5 & 1.137 & 0.180 & 39.685 & 1 & 0.000 & 3.116 \\
Constant & -2.102 & 1.184 & 3.152 & 1 & 0.076 & 0.122 \\
\hline
\end{tabular}

In the final fitting results, four variables were selected (X4 is not significant, and thus to is discarded). The equation is:

$$
\operatorname{Logit}(P)=-2.102+0.742 X_{1}-0.368 X_{2}+1.137 X_{5}
$$

As for the results of regression analysis, "health management" has no significant impact on residents' willingness to sign contracts, while "service level", "medical guidance", "derivative services" and "future development" are important factors that affect residents' willingness to sign contracts.

The reason is that with the rapid development of the Internet, it is extremely easy to obtain all kinds of health care information, so residents' demand for this aspect is not significant. However, "service level", "medical guidance" and "derivative services" directly affect residents' service satisfaction and signing intention, while "future development" represents residents' expectations for the future of the system.

\section{Conclusion and Suggestions}

\subsection{Conclusion}

On the basis of the above research and analysis, it can be demonstrated that Hangzhou has a good overall signing rate for family doctors, with a signing rate of over 30\% ranking top in the whole country. However, there is still the problem of incomplete and unbalanced signing. The signing rate in central urban areas and active communities is relatively good, while the residents in surrounding urban areas and inactive communities know less about the family doctor contract system and the signing rate is rather low. As for the satisfaction with family doctors on contract services, it is found that the "service level" needs to be improved according to the satisfaction matrix in Fig. 1, the program that needs to be improved is "waiting time", and the programs that need to be improved urgently are "medical level", "appointment referral" and "service items". According to logistic regression analysis, there are four factors that significantly affect residents' willingness to sign contracts: "service level", "medical guidance", "derivative services" and "future development".

\subsection{Countermeasures and Suggestions}

Based on the problems found in the above survey and analysis, targeted suggestions are put forward, hoping to effectively improve the satisfaction of the family doctor service system and the signing rate of residents.

4.2.1 Enhance the Publicity and Guidance of Policies

In view of the serious imbalance in the signing rate in different urban areas and communities, the government instructions, indicators and guidance are the most effective and direct means., the Government should raise the attention of district leaders and community workers though policy means so that they can fully disseminate the 
functions and benefits of the system, and then residents can understand the benefits of family doctors to them, see real effects, and increase their confidence in family doctors, and then their concept of medical treatment can be gradually guided to change. Family doctors should also patiently explain to residents the services that the system can provide so that residents can have a full and thorough understanding of the system. In this way, the signing rate of residents can be effectively improved.

4.2.2 Raise the Medical Level of Family Doctors

One of the important reasons why residents' signing rate and satisfaction need to be improved is that the medical level of family doctors is far from that of traditional hospitals. Therefore, it is particularly important to raise their medical level, which can be upgraded through: 1. family doctors and community hospitals can link and pair to upgrade medical equipment; 2 . establish and improve the education and training system for general practitioners, and use regional advantages to strengthen the training of general practitioners so as to improve the comprehensive quality of family doctors; 3 . establish and improve the human resources management mechanism of family doctors, and perfect the team with general practitioners as the core and nurses, public health personnel, neighborhood service personnel and volunteers as the auxiliary, so as to comprehensively raise their medical level in such three aspects.

4.2.3 Improve the Family Doctor Treatment System

In the satisfaction matrix, "appointment referral", "waiting time" and "service items" are located in the third and fourth quadrants and need to be improved urgently. The system can be improved with family doctor APP on the basis of "Internet plus" idea. Residents can report their problems and services needed through APP, and the background makes scientific and reasonable arrangements according to the time of the family doctor, so that each patient can receive the service as soon as possible. For questions that only need text and voice replies, they can be transferred to other free family doctors other than the contracted doctors for answers, which could make full use of resources, greatly improve the efficiency of consultation, and save residents' time. The establishment of a complete medical treatment system through the combination of online and offline can greatly improve residents' satisfaction with the system.

4.2. 4 Improve the Assessment and Incentive System

Family doctors are the core of the whole contract system. Ensuring the service quality and attitude of family doctors is crucial to the sustainable development of the system. Therefore, it is necessary to establish a scientific and reasonable assessment mechanism, with the third-party organization taking residents' satisfaction as the core and conducting assessment of the service attitude, service effect and the feeling of the medical treatment process, so as to ensure the fairness and justice of the assessment. The results of the assessment can be properly publicized and linked to the salaries of family doctors, which can promote the competition among family doctors and promote their service quality.

\section{References}

Albert SM, Shevchik GJ, Paone S, et al. Internet-based medical visit and diagnosis for common medical problems: experience of first user cohort[J]. Telemedicine Journal \& E Health the Official Journal,2011,17(4):304-308.

Bindman AB, Majeed A, et al. Primary care in the United States: organization of primary care in the United States [J]. British Medical Journal,2003,326(7390):631-634.

Qin Dong,Zhen Yuan,GuoQing Ming. Discussion on the integrated intelligent medical service Model of medical maintenance [J]. Health economics Research,2015,7:51-53.

Jiaoling Huang,Yiming Zhang,Shanshan Liu, et al. Analysis on the demand and influencing factors of family doctor contract service in Hongkou District, Shanghai [J]. Health policy research in China,2019,12(08):5461.

LiQun Liu. Promote family doctor signing service to strengthen the construction of the classification system [J]. Chinese General Practice,2018,21(01):1-4.

Lapcević M, Dimitrijević I, Ristić J, et al. Factors influencing general practitioners and specialists of general practice to declare in favor of accepting the role of family doctors [J]. Srpski Arhiv Za Celokupno Lekarstvo,2006,11:128.

Zhijie Song,Beibei Shang,Yan Guo, et al. Research on the Construction of resource integration Service System of "Internet + Community Family Doctor'[J]. Health economics Research,2018,3:32-35.

Peifang Wang,Xiaocui Liu,Zhenzhen He. Promotion of family doctor contract service strategy from the perspective of incentive compatibility theory [J]. Health vocational education,2018, 36(03):147-149.

Hang Zhou,Liang Liu,Zheng Fei, et al. Recognition and satisfaction analysis of graded diagnosis and treatment of medical staff based on importance matrix [J]. World's latest Medical Information Digest,2018,18(83):21-23.

Xueyan Zhang,Jinshui Chen, Yong Chen, et al. A Survey on the Satisfaction of family doctors in Jiangsu Province [J]. Preventive medicine,2020,32(01):74-76. 